\title{
Colonic Metastasis Presenting as an Intraluminal Fungating Mass 8 Years After Surgery for Ovarian Cancer
}

\author{
Jeong Rye Kim, Bong Man Kim, You Me Kim, Won Ae Lee ${ }^{1}$, Hwan Namgung ${ }^{2}$ \\ Departments of Radiology, ${ }^{1}$ Pathology, and ${ }^{2}$ Surgery, Dankook University College of Medicine, Cheonan, Korea
}

We report a case of colonic metastasis from ovarian cancer presented as an intraluminal fungating mass mimicking primary colon cancer 8 years after surgery for ovarian cancer. A 70-year-old woman presented with constipation. She had undergone an extended total abdominal hysterectomy with bilateral salpingo-oophorectomy for an ovarian papillary serous cystadenocarcinoma 8 years earlier. Colonoscopy showed a large fungating mass $10 \mathrm{~cm}$ from the anal verge that was suspected to be colorectal cancer. A computed tomography scan showed a bulky intraluminal fungating mass in the rectosigmoid junction. After a lower anterior resection and a pathologic diagnosis, a diagnosis of a papillary serous adenocarcinoma due to metastasis from an ovarian tumor was made for this patient.

Keywords: Colonic neoplasms; Neoplasm metastasis; Ovarian neoplasms

\section{INTRODUCTION}

Epithelial ovarian carcinomas accounting for $85 \%$ to $90 \%$ of ovarian cancers show aggressive behavior [1]. Epithelial ovarian carcinomas tend to spread as direct invasion, and intraperitoneal seeding, and rarely as hematogenous metastasis [2]. If colonic metastasis from ovarian cancer presents as an intraluminal fungating mass, differentiating it from the primary colon cancer can be difficult. This report describes the case of a patient who had undergone surgery for advanced ovarian cancer and had done well for 8 years, but then presented with a metastasis on the rectosigmoid junction as an intraluminal colonic mass.

\section{CASE REPORT}

A 70-year-old woman presented with constipation in August 2013. Colonoscopy had been performed at an outside institution.

Received: December 24, 2014 - Accepted: April 16, 2015

Correspondence to: Hwan Namgung, M.D.

Departments of Surgery, Dankook University College of Medicine,

119 Dandae-ro, Dongnam-gu, Cheonan 31116, Korea

Tel: +82-41-550-3929, Fax: +82-41-556-3878

E-mail: gsnamgung@dankook.ac.kr

(C) 2015 The Korean Society of Coloproctology

This is an open-access article distributed under the terms of the Creative Commons Attribution NonCommercial License (http://creativecommons.org/licenses/by-nc/3.0) which permits unrestricted noncommercial use, distribution, and reproduction in any medium, provided the original work is properly cited.
A fungating mass of $6 \mathrm{~cm}$ in size was observed $10 \mathrm{~cm}$ from the anal verge and was thought to be colorectal cancer (Fig. 1). A biopsy was performed, and the pathologic diagnosis was a metastatic papillary serous adenocarcinoma. The patient's serum cancer antigen 125 (CA-125) was slightly elevated $(63.2 \mathrm{U} / \mathrm{mL}$; reference, $0-55 \mathrm{U} / \mathrm{mL}$ ), but her serum carcinoembryonic antigen (CEA) was normal (1.5 ng/mL; reference, $0-7 \mathrm{ng} / \mathrm{mL})$. The computed tomography (CT) scan showed a bulky intraluminal fungating mass involving the distal sigmoid colon and the proximal rectum, as well as several small lymph nodes in the superior rectal, left common iliac, and para-aortic nodal stations. Positron emission tomography-CT revealed a high fluoro-deoxyglucose uptake in the colonic mass and in the regional and the para-aortic lymph nodes, suggesting metastatic lymphadenopathy (Fig. 2).

The patient had undergone an extended total abdominal hysterectomy with a bilateral salpingo-oophorectomy for ovarian cancer in June 2005. Pathologic examination revealed a papillary serous cystadenocarcinoma in the right ovary. The tumor involved the contralateral ovary, the bilateral mesosalpinges, and the pelvic peritoneum, and according to the International Federation of Gynecology and Obstetrics staging system, the tumor was stage IIIB. After surgery, the patient was treated with six cycles of paclitaxel, and for eight years, no evidence of recurrence or metastasis was observed. The patient's serum CA 125 level had been $24.6 \mathrm{U} / \mathrm{mL}$ before the first surgery and remained in the normal range until May 2012.

The patient underwent a lower anterior resection with lymph 
Volume 31, Number 5, 2015

node dissection for the regional and the para-aortic lymph nodes. A 6-cm intraluminal mass was noted in the rectosigmoid junction with no evidence of a peritoneal seeding nodule in the pelvic and the abdominal cavities. Macroscopically, the mucosa revealed a huge fungating tumor mimicking the primary colon cancer (Fig. 3). Microscopically, the tumor was an adenocarcinoma with papillary and glandular architectures (Fig. 4). The tumor had infiltrated through the mucosa to the subserosa. Histology data from the previous ovarian cancer were reviewed and were found to be identical to histology data for the present colon tumor. Immunohistochemically, both the ovarian and the colon cancers were diffusely positive for cytokeratin 7 (CK7) and estrogen receptor (ER), but negative for CK20 and mucin 2 (MUC2). The histological and

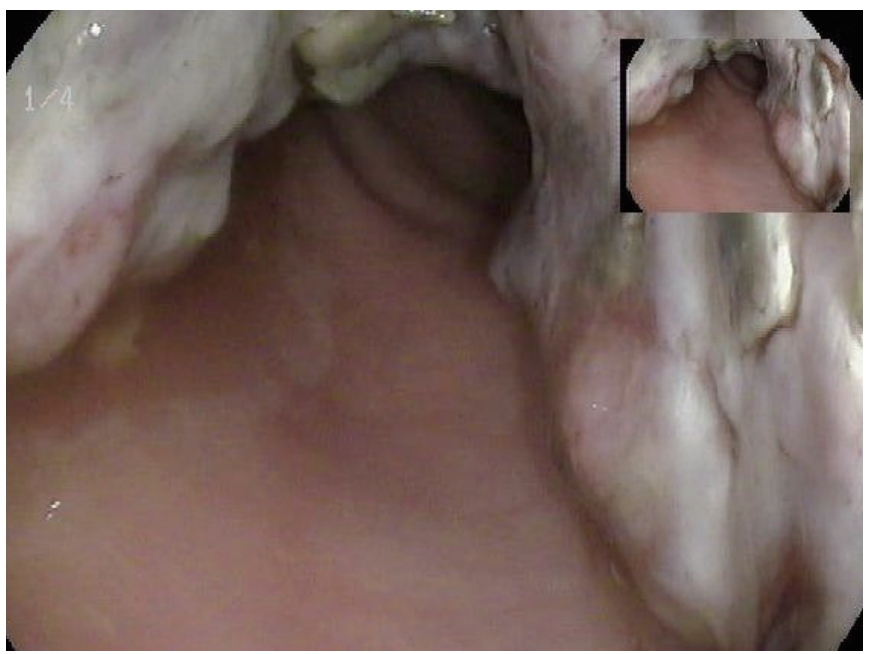

Fig. 1. Colonoscopy shows a large intraluminal fungating mass at the rectosigmoid junction of the colon about $10 \mathrm{~cm}$ from the anal verge.

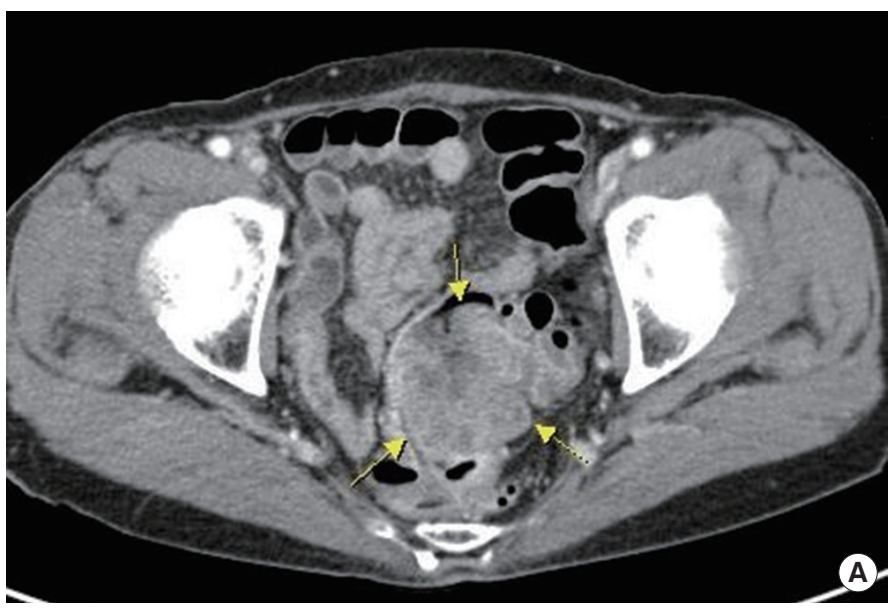

(A)

Fig. 2. (A) The computed tomography (CT) image shows a heterogeneous enhanced mass in the rectosigmoid junction of colon. (B) The colonic mass shows a high fluoro-deoxyglucose uptake on the positron emission tomography-CT image. 


\section{Coloproctology Jeong Rye Kim, et al.}
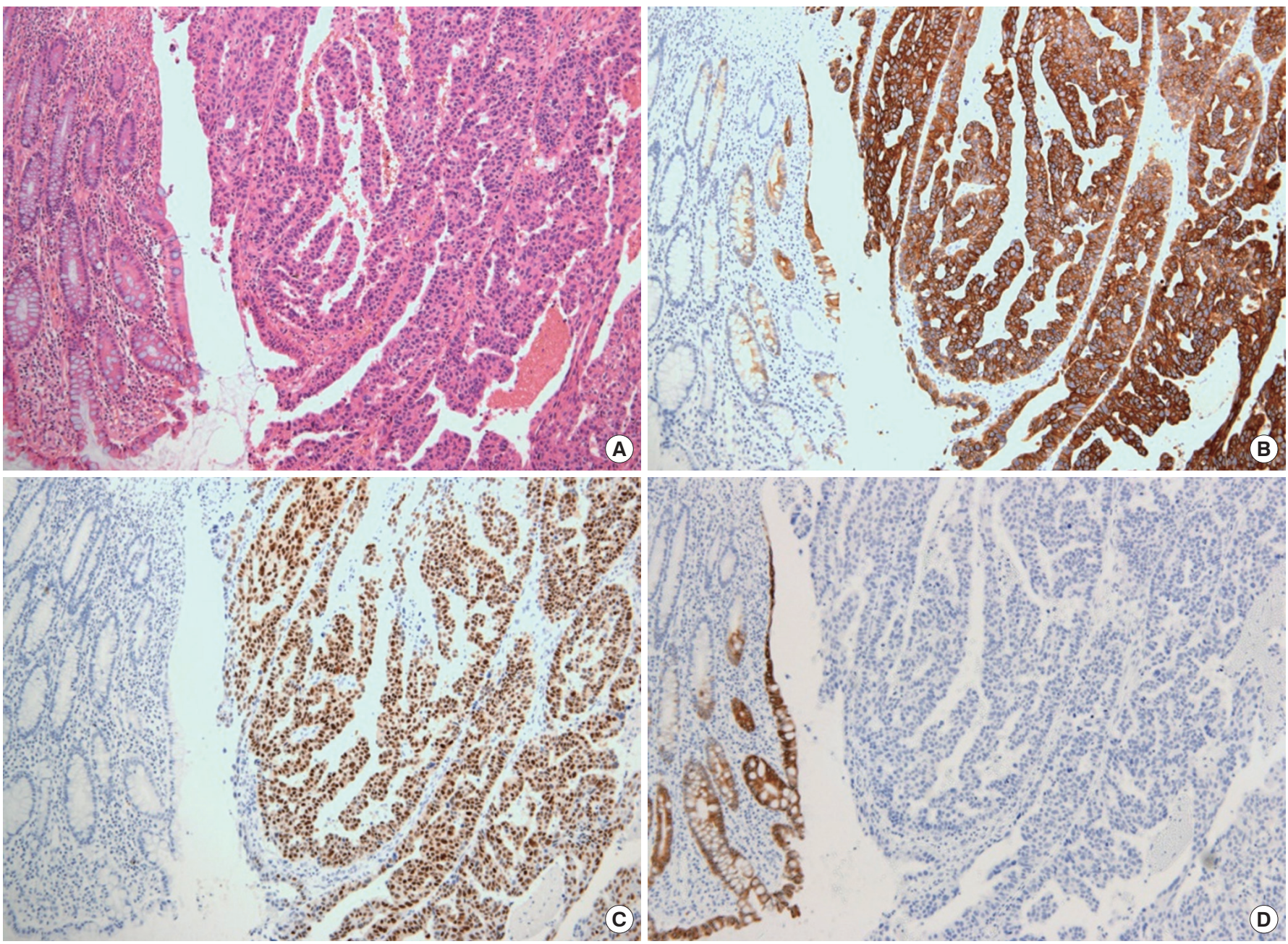

Fig. 4. Colon tumor. (A) Microscopically, the tumor is an adenocarcinoma with papillary and glandular architectures (H\&E, $\times 100)$. The tumor cells are diffusely positive for cytokeratin 7 (CK7) (B) and estrogen receptor (C) and negative for CK20 (D) (immunohistochemistry, $\times 100)$.

\section{DISCUSSION}

Colonic metastases are classified by their mode of dissemination as follows: direct invasion from a contiguous or a noncontiguous primary tumor, intraperitoneal seeding, and embolic metastases [2]. Colonic metastasis from an ovarian epithelial neoplasm usually occurs by direct invasion or intraperitoneal seeding. Therefore, mostly the colonic serosa is initially affected, followed by bowel wall infiltration in an inward direction $[3,4]$. Colonic metastases from ovarian cancer can present as tethering, angulation, and extrinsic mass effects [5]. Zighelboim et al. [3] reported a case of recurrence of an epithelial ovarian carcinoma as an intraluminal bowel lesion with serosal sparing. It was considered to be the result of hematogenous dissemination of tumor cells with infiltration of the submucosal capillary network.

Recurrence of ovarian cancer as colonic metastasis after several years of being disease free is rare. Shibahara et al. [6] reported a case of one patient who underwent surgery to remove a papillary serous adenocarcinoma of both ovaries and then presented with colon metastasis 20 years later. Although the disease-free duration for the patient in the present case was shorter than that of the patient in the case described by Shibahara et al. [6], considering the aggressiveness of ovarian cancer, the emergence of metastasis after eight years is interesting. Kohyama et al. [7] reported another rare case of colon metastasis from ovarian cancer that presented with a protruding colonic mass with intussusception six years after surgery for ovarian cancer.

As in this case, if the colonic metastasis from ovarian cancer presents as an intraluminal fungating mass, a differential diagnosis of colonic metastasis and primary colon cancer based on the macroscopic appearance will be difficult. In such cases, serum CA 125 and CEA levels can be helpful for the differential diagnosis. 
Takahashi et al. [8] reported that in patients with a malignant ovarian tumor, $81.3 \%$ had positive serum CA 125 . However, in patients with a serous cystadenocarcinoma, 100\% showed positive serum CA 125.

Immunohistochemical studies for CK7 and CK20 can be very helpful to differentiate the origin of the tumor. In the study by Loy et al. [9], a CK7 positive/CK20 negative immunophenotype was $100 \%$ specific in differentiating a primary ovarian carcinoma from a metastatic colonic carcinoma. A CK7 negative/CK20 positive immunophenotype was $99 \%$ specific in differentiating a metastatic colonic carcinoma from a primary ovarian carcinoma [9]. Although these are less specific than CK immunostaining, positivity for CA 125, ER, and progesterone receptor immunostainings favor a primary ovarian carcinoma. In contrast, positivity for MUC2 and caudal-type homeobox 2 favors a primary colon cancer. In the present case, both ovarian and colon tumors were diffusely positive for CK7 and ER but completely negative for CK20 and MUC2, supporting both tumors being primary ovarian tumors.

In the past, a bowel resection in a sleeve fashion without a mesenteric resection has been shown to be an effective method of cytoreduction. However, O'Hanlan et al. [10] suggested that surgeons should consider a resection involving the bowel to have a 2to $5-\mathrm{cm}$ longitudinal negative margin with a wedge of mesentery, including the paracolic and intermediate level nodes, which is similar to the current standards for a resection of a primary bowel carcinoma. They recognized that ovarian cancer tended to spread longitudinally along the bowel wall's lymphatic channels into mesenteric nodes. After debulking surgery, a combination of a platinum compound (cisplatin or carboplatin) and taxane (paclitaxel or docetaxel) has been the standard approach for chemotherapy [1].

Distinguishing colonic metastasis from ovarian cancer and primary colon cancer may be difficult. In the case of a patient who presents with an intraluminal colonic mass with a history of ovarian cancer, having a high clinical suspicion of colonic metastasis from ovarian cancer is important. A biopsy followed by appropriate immunochemical staining is required for confirmation.

\section{CONFLICT OF INTEREST}

No potential conflict of interest relevant to this article was reported.

\section{REFERENCES}

1. American Cancer Society. Ovarian cancer [Internet]. Atlanta (GA): American Cancer Society; c2013 [cited 2014 Jul 21]. Available from: http://www.cancer.org/acs/groups/cid/documents/ webcontent/003130-pdf.pdf.

2. Meyers MA, McSweeney J. Secondary neoplasms of the bowel. Radiology 1972;105:1-11.

3. Zighelboim I, Broaddus R, Ramirez PT. Atypical sigmoid metastasis from a high-grade mixed adenocarcinoma of the ovary. Gynecol Oncol 2004;94:850-3.

4. Reed E, Zerbe CS, Brawley OW, Bicher A, Steinberg SM. Analysis of autopsy evaluations of ovarian cancer patients treated at the National Cancer Institute, 1972-1988. Am J Clin Oncol 2000;23: 107-16.

5. Gore RM, Levine MS. Textbook of gastrointestinal radiology. 3rd ed. Philadelphia: Elsevier Saunders; 2008.

6. Shibahara K, Endo K, Ikeda T, Sakata H, Sadanaga N, Morita M, et al. Colon metastasis 20 years after the removal of ovarian cancer: report of a case. Surg Today 2009;39:153-6.

7. Kohyama M, Takesue Y, Ohge H, Sakashita M, Murakami Y, Sueda T. A case of colon metastasis from ovarian cancer presented with intussusception. J Japan Surg Assoc 2005;66:2767-71.

8. Takahashi K, Shibukawa T, Moriyama M, Shirai T, Kijima S, Iwanari $\mathrm{O}$, et al. Clinical usefulness and false-positive results of $\mathrm{CA}$ 125 as a tumor marker of ovarian cancer: a study on 674 patients. Jpn J Surg 1986;16:305-10.

9. Loy TS, Calaluce RD, Keeney GL. Cytokeratin immunostaining in differentiating primary ovarian carcinoma from metastatic colonic adenocarcinoma. Mod Pathol 1996;9:1040-4.

10. O'Hanlan KA, Kargas S, Schreiber M, Burrs D, Mallipeddi P, Longacre $\mathrm{T}$, et al. Ovarian carcinoma metastases to gastrointestinal tract appear to spread like colon carcinoma: implications for surgical resection. Gynecol Oncol 1995;59:200-6. 